\title{
Análise Micro-Histológica da Composição Botânica de Misturas Preestabelecidas, Submetidas ao Processo de Digestão In Vitro ${ }^{1}$
}

\section{Maristela de Oliveira Bauer ${ }^{2}$, José Alberto Gomide ${ }^{3}$, Eldo Antônio Monteiro da Silva ${ }^{4}$, Adair José Regazzi ${ }^{5}$, José Franklim Chichorro 6}

\begin{abstract}
RESUMO - A composição botânica de misturas preestabelecidas de seis espécies forrageiras foi estimada após diferentes tempos de digestão in vitro e comparadas com a composição real. Três misturas de seis espécies, com participações conhecidas, foram constituídas nos períodos chuvoso e seco e examinadas pela técnica micro-histológica. O período de amostragem interferiu na estimativa de apenas uma das misturas. Apesar das diferenças estatísticas entre a composição real das misturas e suas estimativas, nos períodos, seus índices de similaridade foram superiores a $85 \%$. O tempo de digestão influenciou nas estima-tivas das misturas, dependendo da porcentagem de participação das espécies. As estimativas foram idênticas para as três misturas após 48 e 72 horas de digestão. A digestão das amostras pode melhorar ou piorar as estimativas, dependendo da porcentagem de participação e da resistência das espécies. O processo digestivo não deve ser considerado como único fator de variação quando se utiliza esta técnica, que pode ser considerada robusta, uma vez que os índices de similaridade entre os períodos, o tempo de digestão e a composição real foram superiores a 75\%.
\end{abstract}

Palavras-chave: composição real, índice de similaridade, técnica micro-histológica

\section{Microhistological Analysis of the Botanical Composition of Pre-Established Mixtures, Submitted to the In Vitro Digestion Process}

\begin{abstract}
The botanical composition of pre-established mixtures of six forages species was estimated after in vitro digestion times and compared with the real composition. Three mixtures of six species of known participations were made up in the rainy and dry seasons and estimated through the microhistological technique. The sampling season interfered with the estimates of only one of the mixtures. In spite of the statistical differences between the real composition of the mixtures and their estimates, the similarity indices for the periods were superior to $85 \%$. The digestion time influenced mixtures estimates, according to the percentage of the species participation. The three mixtures estimates were identical after 48 and 72 hours of digestion. The digestion of the samples either improved or worsened the estimates, according to the percentage of participation and to species resistance. The digestion process must not be considered as the only factor of variation when this technique is utilized, since the similarity indices between the periods, the digestion time and the real composition were superior to $75 \%$.
\end{abstract}

Key Words: real composition, similarity index and microhistological technique

\section{Introdução}

A análise histológica de fragmentos de plantas em amostras de conteúdo estomacal foi descrita por Baumgartner \& Martin (1939), os pioneiros nessa técnica de determinação do hábito alimentar de animais herbívoros. Mais tarde, Dusi (1949) adaptou essa técnica para análise fecal. No entanto, as primeiras verificações básicas foram realizadas por Sparks \& Malechek (1968).

Com a preocupação básica de verificar a acurácia da estimativa da composição em peso de uma mistura de gramíneas e ervas, Sparks \& Malechek (1968) verificaram que a porcentagem em peso poderia ser predita pela densidade relativa e que a técnica para aquela situação era acurada. O preparo das amostras envolveu secagem, moagem e peneiramento, para reduzir todos os fragmentos a um mesmo tamanho. Dessas amostras, foram montadas lâminas, observando-se 20 campos microscópicos e anotados os fragmentos epidérmicos presentes em cada campo. Assim, foi calculada a porcentagem de freqüência de cada espécie e convertida, pela tabela de Fracker \& Brischle (1944), para densidade relativa. Considerouse cada campo microscópico como uma unidade de amostragem e que os microfragmentos das plantas

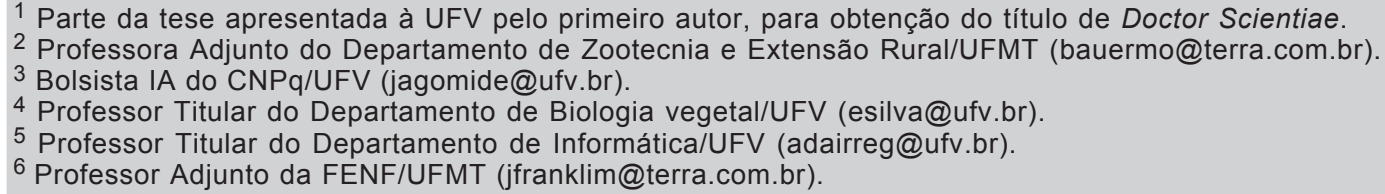


estavam distribuídos ao acaso, todos apresentando o mesmo tamanho e a mesma densidade.

Matematicamente, Johnson (1982) provou ser aceitável a conversão da freqüência em densidade relativa para estimar a composição em peso. Entretanto, Holechek \& Gross (1982b) consideraram desnecessária essa conversão, pois ela aumenta a demanda de tempo e pode resultar em perdas de exatidão.

Apesar de os autores pioneiros terem encontrado relação de 1:1 entre a composição real e a estimada, é possível encontrar na literatura resultados contraditórios. Em geral, alguns componentes são subestimados em relação aos outros.

Westoby et al. (1978) listaram uma série de erros que podem ser cometidos quando se utiliza a técnica micro-histológica, como: uma espécie pode render partículas de tamanhos diferentes; uma espécie, quando sujeita à digestão, pode apresentar proporções diferentes; as espécies podem conter diferentes proporções de fragmentos identificáveis; por exemplo, na identificação de gramíneas, apenas a epiderme é utilizada, enquanto, nas demais categorias, o padrão das nervuras pode auxiliar; a facilidade de identificação pode variar entre os materiais identificáveis; o material pode ser identificado erroneamente; o analista pode adquirir o hábito de procurar a espécie no campo microscópico e, com isso, superestimá-la; o fato de não se saber a qual espécie pertence o material identificável, tornando-o, assim, não-identificável; e se uma espécie estiver sujeita à subestimativa, as demais serão superes-timadas e vice-versa. Dessa forma, a técnica micro-histológica está sujeita a uma série de considerações, e a aceitação dessas considerações é importante para sua utilização.

A proporção de fragmentos epidérmicos identificáveis, por exemplo, pode ser afetada pelas técnicas de preparo das amostras (Antony \& Smith, 1974; Holechek, 1982). O uso de substâncias clareadoras (Williams, 1969; Hinnant \& Kothmann, 1988) e de soluções de hidróxido de sódio (Vavra \& Holechek, 1980) melhora a visualização do fragmento, por remover os pigmentos. Todavia, os fragmentos de algumas espécies podem ser destruídos por essas substâncias (Holechek, 1982). Sem dúvida, o problema da fragmentação diferenciada pode ser resolvido com o peneiramento das amostras durante o preparo das lâminas microscópicas (Hansen et al., 1973).

Considerando o tempo gasto para o preparo das lâminas microscópicas e para identificação dos frag- mentos epidérmicos, Holechek \& Vavra (1981) determinaram que cinco lâminas microscópicas por amostra e 20 campos de observação por lâmina seriam suficientes para estimar com exatidão as espécies que participam com $20 \%$ ou mais, em peso na dieta. Para as espécies com menor participação, há necessidade de número muito maior de lâminas.

Holechek et al. (1982a) não observaram efeito das técnicas de preparo das amostras na determinação da composição botânica, mas verificaram variação nas estimativas entre os observadores. A necessidade de um treinamento sistemático dos observadores foi realçada por Holechek \& Gross (1982a), pois a estes estão associadas as maiores fontes de erros (Gross et al., 1983; Alipayo et al., 1992). Deve-se destacar também que o peso por unidade de área da epiderme pode variar entre e dentro das espécies, ou seja, em grande parte, esses erros se devem às diferenças na relação entre a freqüência dos fragmentos epidérmicos e a biomassa da planta (Gill et al., 1983; Bartolome et al., 1995). Uma mesma planta pode apresentar relação peso/área diferente, seja pela posição da folha na planta seja pela estação do ano, de acordo com o estádio de maturidade.

Em estudos envolvendo digestão, os fragmentos frágeis e suculentos podem ser excessivamente digeridos e, conseqüentemente, subestimados. Assim, espécies de plantas com parede celular e cutícula mais espessas são mais resistentes (Free et al., 1970; Slater \& Jones, 1971; Voth \& Black, 1973; Samuel \& Howard, 1983). No entanto, Mukhtar \& Hansen (1983) não verificaram diferenças entre as frações fibrosas das plantas e as estimativas; logo, rejeitaram a hipótese de que plantas com baixa porcentagem de parede celular sejam sempre subestimadas pela técnica microhistológica. Segundo Duarte et al. (1992), as espécies podem apresentar diferentes graus de dificuldades para identificação e essas dificuldades ocorrem por causa de suas características epidérmicas e do processo que melhor preserve a integridade da epiderme.

Alguns autores sugerem o uso de fatores de correção para evitar as possíveis diferenças geradas não apenas pelo processo digestivo, mas também pela relação peso/área. Esta sugestão não tem unanimidade, pois, quando se inclui uma ou mais espécies na dieta ou ocorrem mudanças em suas proporções, pode haver alteração na digestão individual das espécies. A digestibilidade de partes da planta ou das espécies muda constantemente com o avanço fenológico da 
planta. Portanto, os fatores de correção podem provocar erros significativos (Leslie Jr. et al., 1983). Além disso, Holechek \& Valdez (1985) não recomendam que os dados de ensaios com digestão sejam utilizados como correção para digestão diferencial, porque os métodos de avaliação da digestibilidade apresentam limitações e não devem ser extrapolados, uma vez que existe a necessidade de se obtê-los para todas as espécies de uma área específica de estudo e estação do ano.

Em estudo de composição botânica, Stroup \& Stubbendieck (1983) recomendaram o uso de técnicas multivariadas, tendo em vista a alta correlação entre os percentuais das espécies.

Em decorrência da série de fatores que influenciam a determinação da composição botânica pela técnica micro-histológica, teve-se como objetivo, nesse trabalho, verificar se os tempos de digestão in vitro de amostras colhidas nos períodos chuvoso e seco, alteram a estimativa da participação das espécies de misturas compostas manualmente.

\section{Material e Métodos}

Amostras de lâminas foliares frescas recém-expandidas, correspondentes à última e penúltima posição no perfilho das espécies Melinis minutiflora Pal. de Beauv, Hyparrhenia rufa (Nees) Stapf., Brachiaria decumbens Stapf. e Imperata brasiliensis Trin., e folhas de Medicago sativa L. e Schinus terebentifolius Raddi foram coletadas em seus respectivos canteiros na Agrostologia, área pertencente ao Departamento de Zootecnia da Universidade Federal de Viçosa, durante os períodos chuvoso e seco, e embaladas individualmente em sacolas plásticas. Aquelas relativas ao período das águas foram imediatamente processadas, enquanto as amostras do período seco foram congeladas com nitrogênio líquido e conservadas em congelador a $-80^{\circ} \mathrm{C}$. $\mathrm{O}$ descongelamento deste material ocorreu de forma gradual, pois repassou-se o material para um congelador doméstico a $-20^{\circ} \mathrm{C}$, por duas semanas, em seguida, para geladeira e, finalmente, foi mantido em temperatura ambiente, antes de ser processado.

Para avaliar a influência do grau de digestão na determinação da composição de misturas compostas manualmente, as lâminas foliares de cada espécie foram cortadas individualmente em segmentos de 2 a $4 \mathrm{~mm}$ de comprimento, na porção mediana da lâmina foliar, pesadas e misturadas. Cada mistura envolvia todas as seis espécies em proporções preestabelecidas, conforme Tabela 1.

As misturas foram submetidas à digestão in vitro, segundo metodologia descrita por Tilley \& Terry (1963), com um estádio de digestão. Entretanto, os tempos de digestão utilizados foram 0,48 e 72 horas. Para cada tempo de digestão, utilizaram-se três repetições contendo 1 grama de material.

Ao final da digestão, o material foi liquidificado, por um minuto, lavado e coado em peneira tipo ABNT 140, abertura 0,105 e "Tyler" 150 (Scott \& Dahl, 1980; Duarte et al., 1992). Em seguida, foi submetido ao clareamento em hipoclorito de sódio 5\% (água sanitária doméstica), permanecendo nesta substância pelo tempo necessário, que variou substância variou conforme o material. Após a lavagem, fez-se a montagem das lâminas microscópicas, adicionando-se pequena porção do material e espalhando sobre a lâmina contendo glicerina 50\%. Para vedação, utilizou-se esmalte incolor e confeccionou-se cinco lâminas microscópicas para cada repetição.

As lâminas foram devidamente etiquetadas, mas, durante a leitura, evitou-se conhecer suas identificações, para não viciar as estimativas da composição botânica das misturas. Em cada lâmina microscópica, foram analisados 20 campos de observação, com aumento de 100 vezes (Sparks \& Malechek, 1968; Holechek \& Vavra, 1981). Para cada campo, foram

Tabela 1 - Composição real, em porcentagem de peso verde, dos componentes das misturas $A, B$ e $C$ Table 1 - Real composition, in percentage of green weight, of the components $A, B$ and $C$ mixtures

\begin{tabular}{|c|c|c|c|}
\hline \multirow[t]{2}{*}{$\begin{array}{l}\text { Espécie } \\
\text { Specie }\end{array}$} & \multicolumn{3}{|c|}{$\begin{array}{l}\text { Mistura } \\
\text { Mixture }\end{array}$} \\
\hline & A & B & $\mathrm{C}$ \\
\hline $\begin{array}{l}\text { Capim-gordura } \\
\text { Molassesggrass }\end{array}$ & 30 & 10 & 10 \\
\hline $\begin{array}{l}\text { Capim-sapé } \\
\text { Sapegrass }\end{array}$ & 30 & 10 & 10 \\
\hline $\begin{array}{l}\text { Capim-jaraguá } \\
\text { Jaraguagrass }\end{array}$ & 10 & 30 & 10 \\
\hline $\begin{array}{l}\text { Capim-braquiária } \\
\text { Signagrass }\end{array}$ & 10 & 30 & 10 \\
\hline $\begin{array}{l}\text { Alfafa } \\
\text { Alfalfa }\end{array}$ & 10 & 10 & 30 \\
\hline $\begin{array}{l}\text { Aroeira } \\
\text { Aroeira }\end{array}$ & 10 & 10 & 30 \\
\hline
\end{tabular}


anotadas as presenças dos fragmentos das espécies que se conseguia identificar, determinando-se a freqüência relativa de cada componente, obtida pela seguinte relação:

$\%=\frac{\text { freqüência de cada componente }}{\sum \text { das freqüências de todos os componentes identificados }} \times 100$

conforme Holechek \& Gross (1982b).

Para a análise dos dados utilizou-se procedimento PROC GLM (General Linear Models) do SAS (1990). A análise de variância multivariada (MANOVA) foi utilizada para avaliar a porcentagem de cada componente das misturas $\mathrm{A}, \mathrm{B}$ e $\mathrm{C}$, tomadas nos dois períodos (chuvoso e seco) e antes e após o processo digestivo (tempos de digestão de 0,48 e 72 horas), adotando-se o delineamento inteiramente casualizado no esquema fatorial, com três repetições, por meio do seguinte modelo estatístico:

$$
\begin{gathered}
\mathrm{Y}_{\mathrm{ijkl}}=\mathrm{m}_{1}+\mathrm{P}_{\mathrm{il}}+\mathrm{T}_{\mathrm{jl}}+\mathrm{PT}_{\mathrm{ijl}}+\epsilon_{\mathrm{ijkl}} \\
\operatorname{com} \mathrm{i}=1,2 ; \mathrm{j}=1,2 \text { e } 3 ; \mathrm{k}=1,2 \text { e } 3 ; 1=1,2, \ldots \text { e } 6 .
\end{gathered}
$$

em que $\mathrm{Y}_{\mathrm{ijkl}}=$ observação referente à avaliação k, no período $\mathrm{i}$, no tempo $\mathrm{j}$, da espécie $1 ; \mathrm{m}_{1}=$ média geral da espécie $1 ; \mathrm{P}_{\mathrm{i} 1}=$ efeito do período $\mathrm{i}$, na espécie $1 ; \mathrm{T}_{\mathrm{jl}}=$ efeito do tempo $\mathrm{j}$, na espécie $1 ; \mathrm{PT}_{\mathrm{ijl}}=$ efeito da interação período i e tempo j, na espécie $1 ; \mathrm{e} \in \in_{\mathrm{ijkl}}=$ erro aleatório associado à observação $\mathrm{Y}_{\mathrm{ijkl}}$. Tem-se ainda que os vetores $\underset{\sim \text { ijk }}{\in}=\left[\epsilon_{\mathrm{ijk} 1}, \in_{\mathrm{ijk} 2}, \ldots ., \in_{\mathrm{ijkp}}\right]$, com $\mathrm{p}=6$, têm distribuição multinormal p-dimensional com vetor nulo de médias e uma matriz de covariâncias $\mathrm{S}$ comum a todas as combinações i, j e k. Os vetores $\underset{\sim}{\in} \underset{\text { ijk }}{ }$ correspondentes a diferentes unidades experimentais são independentemente distribuídos.

$\mathrm{O}$ teste $\mathrm{T}^{2}$ de Hotelling (Johnson \& Wichern, 1992) foi utilizado para realização das comparações entre os vetores de médias da composição real e os de médias das composições estimadas de cada tempo de digestão e de cada período, entre os vetores de médias das composições estimadas dos períodos (chuvoso e seco) e entre os vetores de médias das composições estimadas de cada tempo de digestão, para as misturas $\mathrm{A}, \mathrm{B}$ e $\mathrm{C}$.
O índice de similaridade foi calculado pela fórmula de Kulcyznski (Oosting, 1956; Hansen \& Reid, 1975; Smith \& Shandruck, 1979; Alipayo et al., 1992):

$$
\mathrm{IS}_{j \mathrm{k}}=\frac{2 \sum_{\mathrm{i}=1}^{\mathrm{I}} \min \left(\mathrm{P}_{\mathrm{ij}}, \mathrm{P}_{\mathrm{ik}}\right)}{\sum_{\mathrm{i}=1}^{\mathrm{I}}\left(\mathrm{P}_{\mathrm{ij}}+\mathrm{P}_{\mathrm{ik}}\right)} \times 100
$$

em que $\mathrm{IS}_{\mathrm{jk}}=$ índice de similaridade $(\%) ; \mathrm{P}_{\mathrm{ij}}=$ valor percentual do componente $\mathrm{i}$ na dieta $\mathrm{j}$; e $\mathrm{P}_{\mathrm{ik}}=$ valor percentual do componente i na dieta $\mathrm{k}$.

Os índices foram utilizados para se avaliar a similaridade entre a composição real e as composições estimadas de amostras colhidas nos períodos chuvoso e seco, entre a composição real e os tempos de digestão, entre os períodos e entre os tempos de digestão. Segundo Hansen (1971), índices de similaridade iguais ou superiores a $85 \%$ indicam que as amostras são altamente similares.

\section{Resultados e Discussão}

Os vetores de médias das composições percentuais reais das amostras $\mathrm{A}, \mathrm{B}$ e $\mathrm{C}$, com suas respectivas estimativas para os períodos chuvoso e seco, estão apresentados na Tabela 2. Apesar do índice de similaridade da mistura A ter sido de $90 \%$, essa mistura foi a única a apresentar diferença significativa $(\mathrm{P}<0,01)$ entre os períodos. As espécies que mais contribuíram para essa diferença foram os capins sapé e jaraguá (Tabela 2 e 3).

As composições estimadas em ambos os períodos diferiram $(\mathrm{P}<0,01)$ da composição real, para todas as misturas (Tabela 3). Nas misturas B e C, os índices obtidos entre o período seco e a composição real foram superiores àqueles do período chuvoso. É possível que as gramíneas com maior participação (30\%) nas misturas tenham sido subestimadas, gerando índices de 85 e $86 \%$ no período chuvoso, para as respectivas misturas.

A estimativa da mistura A manteve um padrão de similaridade com a composição real (índices de 90\%). Entretanto, esses índices poderiam ter sido superiores a $90 \%$ e o teste $\mathrm{T}^{2}$ de Hotelling acusado nãosignificância se o componente aroeira não tivesse sido superestimado (Tabela 2).

$\mathrm{Na}$ Tabela 4 estão apresentados os vetores de médias da porcentagem de participação das espécies 
Tabela 2 - Vetores de médias das composições percentuais reais das misturas A, B e C, com suas respectivas estimativas para os períodos chuvoso e seco

Table 2 - Mean vectors of the real composition percentage of the mixtures $A, B$ and $C$, with its respective estimates for the rainy and dry seasons

\begin{tabular}{llcc}
\hline $\begin{array}{l}\text { Mistura } \\
\text { Mixture }\end{array}$ & Real & Estimada período chuvoso & Estimada período seco \\
\hline
\end{tabular}

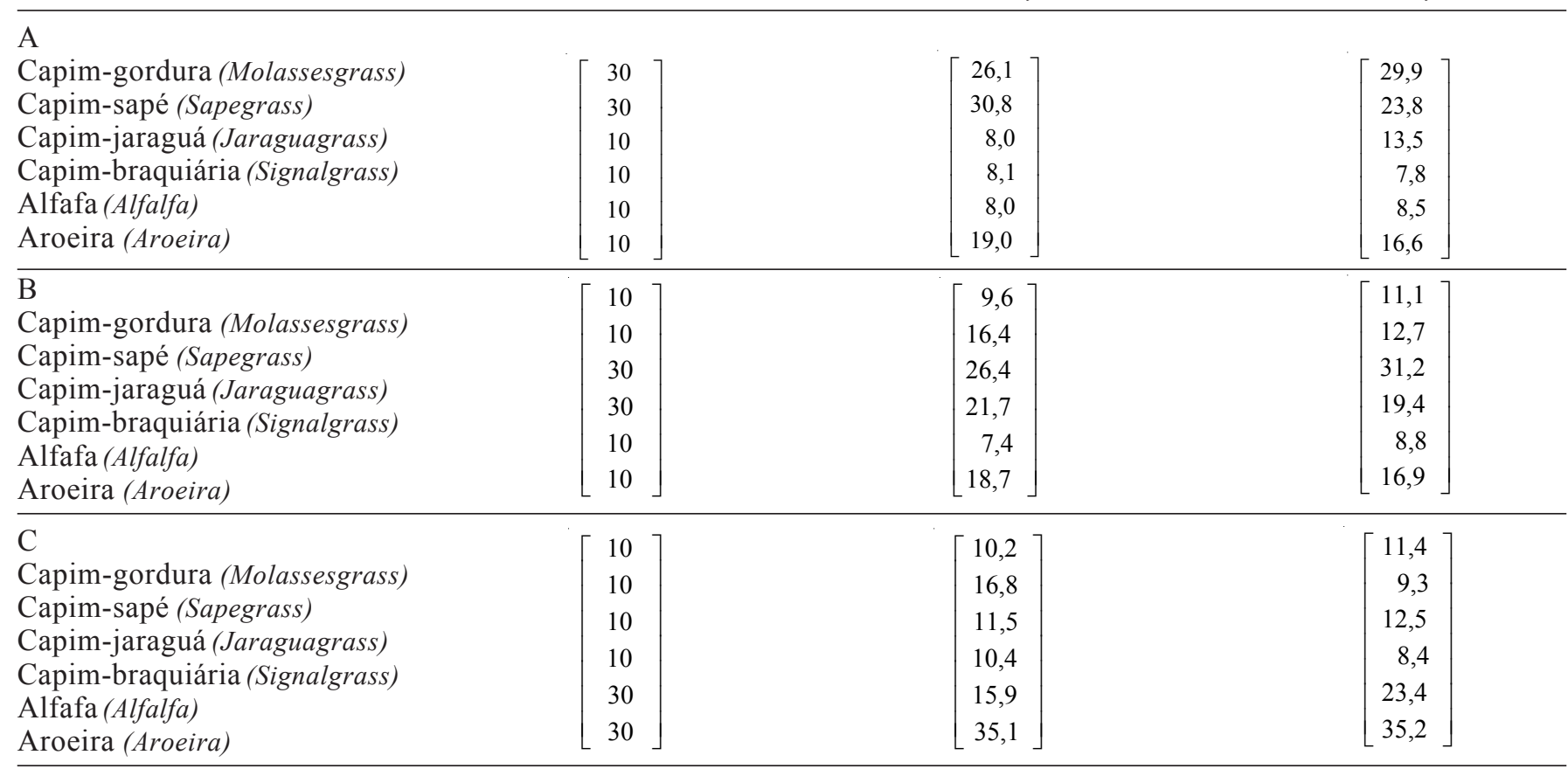

Tabela 3 - Índices de similaridade (\%) e significâncias do teste $\mathrm{T}^{2}$ de Hotelling entre o vetor da composição real das misturas $\mathrm{A}, \mathrm{B}$ e $\mathrm{C}$ e os vetores de médias estimados para os períodos chuvoso (P1) e seco (P2)

Table 3 - Similarity indices (\%) and comparing mean vectors, by Hotelling $T^{2}$ test, of the real composition of the mixtures $A, B$ and $C$ and estimated composition for the rainy $(P 1)$ and dry seasons $(P 2)$

\begin{tabular}{lccc}
\hline Comparação & \multicolumn{3}{c}{$\begin{array}{c}\text { Mistura } \\
\text { Comparixture }\end{array}$} \\
\cline { 2 - 4 } & $\mathrm{A}$ & $\mathrm{B}$ & $\mathrm{C}$ \\
\hline $\mathrm{P} 1$ vs P2 & $90 *$ & $92^{\mathrm{ns}}$ & $90^{\mathrm{ns}}$ \\
Composição real vs P1 & $90 *$ & $85^{*}$ & $86 *$ \\
Real composion vs P1 & & & \\
Composição real vs P2 & $90 *$ & $88^{*}$ & $91 *$ \\
Real composion vs $P 2$ & & & \\
\hline
\end{tabular}

* Diferença significativa $(P<0,01)$ pelo teste $T^{2}$ de Hotelling.

* Significant difference $(P<0.01)$ by Hotelling $T^{2}$ test.

ns - Diferença não-significativa $(P>0,01)$ pelo teste $T^{2}$ de Hotelling. $n s$ - Not significant difference $(P<0.01)$ by Hotelling $T^{2}$ test. na composição real e nas composições estimadas para os tempos de digestão $(0,48$ e 72 horas $)$ das misturas A, B e C. Verificou-se tendência do efeito do processo digestivo sobre a participação das espécies nas misturas, o que pode ser comprovado pelos índices de similaridade e pelas significâncias dos testes estatísticos apresentados nas Tabelas 5 e 6 .

Os resultados do teste $\mathrm{T}^{2}$ de Hotelling e os baixos índices de similaridade, de 68 a 79\%, sugerem discrepância entre as estimativas de 0 e 48 horas e de 0 e 72 horas de digestão (Tabela 5). No entanto, uma vez ocorrido o processo de digestão ( 48 ou 72 horas), passa a não haver diferenças entre as estimativas, o que é confirmado pela não-significância do teste $(\mathrm{P}>0,01)$, para todas as composições, e pelos índices de similaridade, que variaram de 90 a $96 \%$. 
Tabela 4 - Vetores de médias da composição percentual real de A, B e C, com suas respectivas estimativas, antes e após o processo de digestão in vitro

Table 4 - Mean vectors (\%) real composition of $A, B$ and $C$, with its respective estimates before and after the process of in vitro digestion

\begin{tabular}{|c|c|c|c|c|}
\hline $\begin{array}{l}\text { Mistura } \\
\text { Mixture }\end{array}$ & $\begin{array}{l}\text { Real } \\
\text { Real }\end{array}$ & $\begin{array}{c}\text { Estimada sem } \\
\text { digestão } \\
\text { Estimated without } \\
\text { digestion }\end{array}$ & $\begin{array}{l}\text { Estimada após } \\
48 \text { horas digestão } \\
\text { Estimated } 48 \mathrm{~h} \\
\text { digestion }\end{array}$ & $\begin{array}{l}\text { Estimada após } \\
72 \text { horas digestão } \\
\text { Estimated } 72 \mathrm{~h} \\
\text { digestion }\end{array}$ \\
\hline $\begin{array}{l}\text { A } \\
\text { Capim-gordura (Molassesgrass) } \\
\text { Capim-sapé (Sapegrass) } \\
\text { Capim-jaraguá (Jaraguagrass) } \\
\text { Capim-braquiária (Signalgrass) } \\
\text { Alfafa (Alfalfa) } \\
\text { Aroeira (Aroeira) }\end{array}$ & $\begin{array}{l}30 \\
30 \\
10 \\
10 \\
10 \\
10\end{array}$ & {$\left[\begin{array}{r}26,7 \\
17,2 \\
5,6 \\
10,8 \\
21,9 \\
17,8\end{array}\right]$} & {$\left[\begin{array}{r}27,5 \\
33,2 \\
12,0 \\
7,8 \\
1,5 \\
18,0\end{array}\right]$} & {$\left[\begin{array}{r}29,7 \\
31,6 \\
14,7 \\
5,2 \\
1,3 \\
17,5\end{array}\right]$} \\
\hline $\begin{array}{l}\text { B } \\
\text { Capim-gordura (Molassesgrass) } \\
\text { Capim-sapé (Sapegrass) } \\
\text { Capim-jaraguá (Jaraguagrass) } \\
\text { Capim-braquiária (Signalgrass) } \\
\text { Alfafa (Alfalfa) } \\
\text { Aroeira (Aroeira) }\end{array}$ & $\begin{array}{l}10 \\
10 \\
30 \\
30 \\
10 \\
10\end{array}$ & {$\left[\begin{array}{r}9,7 \\
8,7 \\
18,6 \\
26,8 \\
19,8 \\
16,3\end{array}\right]$} & {$\left[\begin{array}{r}10,1 \\
16,5 \\
32,5 \\
21,1 \\
3,3 \\
16,4\end{array}\right.$} & {$\left[\begin{array}{r}11,1 \\
18,3 \\
35,3 \\
13,7 \\
1,0 \\
20,6\end{array}\right.$} \\
\hline $\begin{array}{l}\text { C } \\
\text { Capim-gordura (Molassesgrass) } \\
\text { Capim-sapé (Sapegrass) } \\
\text { Capim-jaraguá (Jaraguagrass) } \\
\text { Capim-braquiária (Signalgrass) } \\
\text { Alfafa (Alfalfa) } \\
\text { Aroeira (Aroeira) }\end{array}$ & $\begin{array}{l}10 \\
10 \\
10 \\
10 \\
30 \\
30\end{array}$ & $\begin{array}{r}10,1 \\
6,4 \\
8,5 \\
10,9 \\
33,8 \\
30,2\end{array}$ & $\begin{array}{l}10,8 \\
16,2 \\
12,6 \\
10,5 \\
12,7 \\
37,2\end{array}$ & $\begin{array}{r}11,5 \\
16,4 \\
14,8 \\
6,8 \\
12,4 \\
38,1\end{array}$ \\
\hline
\end{tabular}

De acordo com a Tabela 6, as estimativas das amostras tomadas no tempo zero de digestão diferi$\operatorname{ram}(\mathrm{P}<0,01)$ da composição real, exceto a estimativa da composição da mistura $\mathrm{C}$.

A diferença entre a estimativa da mistura A e sua composição real no tempo zero de digestão pode ser atribuída às superestimativas da aroeira e do capimsapé (Tabela 4). Em amostras tomadas após 48 e 72 horas de digestão, foi registrada melhoria na identificação do capim-sapé, o que pode ser comprovado pelo aumento no índice de similaridade entre a composição real e as estimativas. Entretanto, esses índices não atingiram patamares mais elevados, em decorrência da subestimativa da alfafa.

Quanto às comparações realizadas em amostras da mistura $\mathrm{B}$, as diferenças podem ser atribuídas ao capim-jaraguá, à alfafa e ao capim-braquiária. $\mathrm{O}$ tempo de digestão de 72 horas teve maior efeito sobre a estimativa dessa mistura que os demais, pois seu índice de similaridade, em relação à composição real, foi $9 \%$ menor.
As quedas nos índices de similaridade da mistura $\mathrm{C}$ refletem a fragilidade da alfafa e a melhoria na identificação da aroeira ao processo de digestão, ocasionando sub e superestimativa dessas plantas, respectivamente.

A estimativa da participação do capim-gordura em todas as misturas foi a única a não variar com o processo digestivo, apresentando valores muito próximos, especialmente os da composição real. Os valores da participação da aroeira foram todos superestimados em relação à composição real, com exceção da estimativa da composição C no tempo 0 (Tabela 4).

Quando a participação dos capins sapé e jaraguá foi de $30 \%$ na composição real, essas espécies foram subestimadas no tempo de digestão 0 e seus valores estimados se aproximaram do valor real após 48 e 72 horas de digestão. Por outro lado, quando suas participações foram de $10 \%$ na composição real, houve superestimativa dos valores nos referidos tempos de digestão e esses seguiram um padrão de aumento nos percentuais com o aumento no tempo de digestão. 
Tabela 5 - Índice de similaridade (\%) e significâncias do teste $\mathrm{T}^{2}$ de Hotelling entre os vetores de médias dos tempos de digestão 0, 48 e 72 horas, para as misturas $\mathrm{A}, \mathrm{B}$ e $\mathrm{C}$

Table 5 - Similarity index (\%) and comparing mean vectors, by Hotelling $T^{2}$ test, of between digestion times 0 , 48 and 72 hours, for the mixtures $A, B$ and $C$

\begin{tabular}{|c|c|c|c|}
\hline \multirow[t]{2}{*}{$\begin{array}{l}\text { Comparação } \\
\text { Comparison }\end{array}$} & \multicolumn{3}{|c|}{$\begin{array}{l}\text { Mistura } \\
\text { Mixture }\end{array}$} \\
\hline & A & $\mathrm{B}$ & $\mathrm{C}$ \\
\hline $\begin{array}{l}\text { Tempo } 0 \text { vs Tempo } 48 \\
\text { Time } 0 \text { vs Time } 48\end{array}$ & $77 *$ & $78 *$ & $79 *$ \\
\hline $\begin{array}{l}\text { Tempo } 0 \text { vs Tempo } 72 \\
\text { Time } 0 \text { vs Time } 72\end{array}$ & $73 *$ & $68 *$ & $75 *$ \\
\hline $\begin{array}{l}\text { Tempo } 48 \text { vs Tempo } 72 \\
\text { Time } 48 \text { vs Time } 72\end{array}$ & $95^{\mathrm{ns}}$ & $90^{\mathrm{ns}}$ & $96^{\mathrm{ns}}$ \\
\hline
\end{tabular}

* Diferença significativa $(P<0,01)$ pelo teste $T^{2}$ de Hotelling.

* Significant difference $(P<0.01)$ by Hotelling $T^{2}$ test.

ns - Diferença não-significativa $(P>0,01)$ pelo teste $T^{2}$ de Hotelling. $n s$ - Not significant difference $(P<0.01)$ by Hotelling $T^{2}$ test.

As estimativas do capim-braquiária foram semelhantes à porcentagem real para os tempos 0 e 48 horas de digestão e subestimado para o tempo 72 horas de digestão, quando sua participação foi de $10 \%$ na composição real. Todavia, ao contribuir com $30 \%$ na mistura real, sua participação foi subestimada em todos os tempos de digestão.

A espécie mais prejudicada pelo processo digestivo foi a alfafa, esse fato provocou em todas as misturas uma subestimativa. No tempo zero de digestão, seus valores foram superestimados, principalmente quando sua participação real perfazia $10 \%$.

As estimativas são confiáveis quando há semelhança entre as espécies quanto à cutinização, fragmentação na digestão, taxa de digestão e relação epiderme/volume de tecido de planta. Pelo fato de plantas com características diferentes terem participado da composição das misturas, algumas espécies foram insatisfatoriamente estimadas. A identificação de espécies, seja pela sua facilidade seja pelo seu número de fragmentos produzidos, resulta necessariamente em sua superestimativa e na subestimativa de outras espécies.

A superestimativa do capim-jaraguá em mistura do período seco contradiz o resultado obtido por Bauer (2000), no qual o capim-jaraguá apresentou maior percentual de fragmentos identificáveis em amostras do período chuvoso. Acredita-se que tenha havido efeito compensatório entre os capins jaraguá, sapé e
Tabela 6 - Índice de similaridade (\%) e significâncias do teste $\mathrm{T}^{2}$ de Hotelling entre o vetor da composição real das misturas $\mathrm{A}, \mathrm{B}$ e $\mathrm{C}$ e os vetores de médias estimados para os tempos de digestão 0, 48 e 72 horas

Table 6 - Similarity index (\%) and comparing mean vectors, by Hotelling $T^{2}$ test, of between the real composition of the mixtures $A, B$ and $C$ and estimated composition in the digestion times 0,48 and 72 hours

\begin{tabular}{lccc}
\hline Comparação & \multicolumn{3}{c}{$\begin{array}{c}\text { Mistura } \\
\text { Comparixture }\end{array}$} \\
\cline { 2 - 4 } & A & B & C \\
\hline $\begin{array}{l}\text { Composição real vs Tempo 0 } \\
\text { Real composition vs Time 0 }\end{array}$ & $80 *$ & $84 *$ & 95 ns \\
$\begin{array}{l}\text { Composição real vs Tempo 48 } \\
\text { Real composition vs Time 48 }\end{array}$ & $87 *$ & $84 *$ & $83 *$ \\
$\begin{array}{l}\text { Composição real vs Tempo } 72 R \\
\text { Real composition vs Time 72 }\end{array}$ & $86 *$ & $75 *$ & $79 *$ \\
\hline
\end{tabular}

* Diferença significativa $(P<0,01)$ pelo teste $T^{2}$ de Hotelling.

* Significant difference $(P<0.01)$ by Hotelling $T^{2}$ test.

ns - Diferença não-significativa $(P>0,01)$ pelo teste $T^{2}$ de Hotelling. $n s$ - Not significant difference $(P<0.01)$ by Hotelling $T^{2}$ test.

braquiária, nas estimativas de amostras de mistura do período chuvoso, pois estas duas últimas espécies foram subestimadas neste período, para todas as misturas.

A extensão da digestão de espécies individuais pode ser alterada quando são incluídas numa dieta quando outras espécies estão presentes e isto acarreta mudanças na participação real das espécies (Holechek \& Valdez, 1985). As plantas respondem diferentemente ao processo digestivo. Enquanto umas podem se tornar não-identificáveis, outras, apesar de identificáveis, podem se fragmentar de forma diferenciada e, em vista da proporção das espécies, pode ter a contagem dos fragmentos alterada quando utilizada como base para determinação. Isto é muito comum quando plantas com diferentes características epidérmicas estão envolvidas.

Quando se trabalha com animais, normalmente encontra-se diferenças de composição entre os períodos (Bauer et al., 1998), o que se deve à seletividade dos animais. Além de os animais tenderem a selecionar as espécies mais suculentas, a disponibilidade de forragem na área pastejada varia.

Os índices de similaridade expressos na Tabela 3 evidenciam que todas as espécies presentes na composição real foram detectadas e estimadas a contento (índices superiores a 85\%).

Os tempos de digestão estudados referem-se a alguns pontos do trato digestivo de animais, sem 
considerar a taxa de passagem. As amostras coletadas no tempo zero podem se referir àquelas coletadas em fístulas esofágicas; e as coletadas nos tempos de 48 e 72 horas, às coletadas no rúmen e nas fezes, respectivamente.

Após 48 horas de digestão, as alterações na composição foram mínimas, mas a intensidade dependeu da porcentagem real dos fragmentos de plantas frágeis, no caso, a alfafa. Os fragmentos mais resistentes e aqueles que apresentaram problemas com a pigmentação já nas primeiras 48 horas foram mais bem visualizados por causa do afrouxamento das células, da diminuição da pigmentação e da separação da epiderme, como discutido por Bauer (2000). Em decorrência desses comportamentos antagônicos das espécies que participaram das misturas, houve compensação nas porcentagens de participação das espécies.

Acredita-se que a presença da alfafa e da aroeira foi a principal responsável pelas diferenças nas estimativas das misturas, especialmente quando suas participações reais foram de $10 \%$, independentemente do tempo de digestão, confirmando os achados de alguns autores, que atribuem os erros de estimativa à presença desses tipos de plantas.

Alguns autores (Holechek et al., 1982b) recomendam o uso de fatores de correção para minimizar as discrepâncias causadas pelo processo digestivo, mas a taxa e a extensão da digestão variam constantemente com as partes, os tipos de plantas e o estádio fenológico. A dieta de um animal muda constantemente em termos de proporção de espécies e partes da planta, tornando difícil determinar um fator para correção.

O uso de técnicas de microdigestão, como as utilizadas nesse trabalho, ou de equações de regressão para a determinação dos fatores de correção, pode provocar erros grosseiros quando os resultados são extrapolados. As técnicas de digestão apenas exprimem o potencial de digestão das espécies. No animal, outros fatores estão envolvidos, como o tempo de retenção da partícula e a taxa de passagem, que têm papel preponderante na digestão. Portanto, o uso de equações deve ser restrito para aquelas condições na qual foram obtidas.

Com base nesse trabalho e em dados observados na literatura, recomenda-se que, ao se utilizar a técnica micro-histológica, suas limitações sejam consideradas e que, ao interpretar os resultados obtidos, utilize-se o bom senso, tendo uma idéia do todo sem considerar espécies isoladas.

\section{Conclusões}

Apesar de as diferenças estatísticas entre a composição real das misturas e suas estimativas, os índices de similaridade entre os períodos foram superiores a $85 \%$.

O tempo de digestão influenciou as estimativas das misturas, dependendo da porcentagem de participação das espécies.

As estimativas dos tempos 48 e 72 horas de digestão foram idênticas para as três misturas.

A presença da alfafa e da aroeira reduziu a acurácia das estimativas.

O processo digestivo melhorou as estimativas de espécies mais resistentes.

O processo digestivo não deve ser considerado como único fator de variação quando se utiliza essa técnica, que pode ser considerada robusta, pois os índices de similaridade entre os períodos, o tempo de digestão e a composição real foram superiores a $75 \%$.

\section{Literatura Citada}

ALIPAYO, D.; VALDEZ, R.; HOLECHEK, J.L. et al. Evaluation of microhistological analysis for determining ruminant diet botanical composition. Journal of Range Management, v.45, n.2, p.148-152, 1992.

ANTHONY, R.G.; SMITH, N.S. Comparison of rumen and fecal analysis to describe deer diets. Journal Wildlife Management, v.38, n.3, p.535-540, 1974.

BARTOLOME, J.; FRANCH, J.; GUTMAN, M. et al. Physical factors that influence fecal analysis estimates of herbivore diets. Journal of Range Management, v.48, n.3, p.267270, 1995

BAUER, M.O. Avaliação da técnica microhistológica para estimativa da dieta de ruminantes. Viçosa, MG: Universidade Federal de Viçosa, 2000. 152p. Tese (Doutorado em Zootecnia)-Universidade Federal de Viçosa, 2000.

BAUER, M.O.; NASCIMENTO JR., D.; SILVA, E.A.M.et al. Composição botânica da dieta de bovinos nos relevos côncavo e convexo, em pastagem natural de Viçosa-MG. Revista Brasileira de Zootecnia, v.27, n.1, p.1-8, 1998.

BAUMGARTNER, L.L.; MARTIN, A.C. Plant histology as an aid in squirrel food-habit studies. Journal Wildlife Management, v.3, p.266-268, 1939.

DUARTE, C.M.L.; NASCIMENTO JR., D.; SILVA, E.A.M. et al. Métodos para estimar a composição botânica da dieta de herbívoros. Revista Brasileira de Zootecnia, v.21, n.2, p.279-290, 1992

DUSI, J.L Methods for determination of food habits by plant microtechniques and histology and their applications to cottontail rabbits in Ohio. Journal Wildlife Management, v.13, n.2, p.295-298, 1949.

FRACKER, S.R.; BRISCHLE, H.A. Measuring the local distribution of ribes. Ecology, v.25, n.3, p.283-303, 1944 
FREE, J.C.; HANSEN, R.M.; SIMS, P.L. Estimating dry weights of foodplants in feces of herbivores. Journal of Range Management, v.23, p.300-306, 1970.

GILL, R.B.; CARPENTAR, L.H.; BARTMANN, R.M. et al. Fecal analysis to estimate mule deer diets. Journal Wildlife Management, v.47, p.902-915, 1983.

GROSS, B.D.; MAHGOUB, E.; HOLECHEK, J. Mastication effects on cattle diet determined by microhistological analysis. Journal of Range Management, v.36, n.4, p.475-476, 1983.

HANSEN, R.M. Estimating plant composition of wild sheep diets. First Trans North American Wild Sheep Conference, p.180-85, 1971.

HANSEN, R.M.; PEDEN, D.G.; RICE, R.W. Discerned fragments in feces indicates diet overlap. Journal of Range Management, v.26, n.2, p.103-105, 1973.

HANSEN, R.M.; REID, L.D. Diet overlap of deer, elk, and cattle in southern Colorado. Journal of Range Management, v.28, n.1, p.43-47, 1975.

HINNANT, R.T.; KOTHMANN, M.M. Collecting, drying, and preserving feces for chemical and microhistological analysis. Journal of Range Management, v.41, n.2, p.168-171, 1988.

HOLECHEK, J.L. Sample preparation techniques for microhistological analysis. Journal of Range Management, v.35, n.2, p.267-268, 1982.

HOLECHEK, J.L.; GROSS, B. Training needed for quantifying simulated diets from fragmented range plants. Journal of Range Management, v.35, n.5, p.644-647, 1982a.

HOLECHEK, J.L.; GROSS, B.D.; DABO, S.M. et al. Effects of sample preparation, growth stage, and observer on microhistological analysis of herbivores diets. Journal Wildlife Management, v.46, n.2, p.502-505, 1982a.

HOLECHEK, J.L.; GROSS, B.D. Evaluation of different calculation procedures for microhistological analysis. Journal of Range Management, v.35, n.6, p.721-723, $1982 \mathrm{~b}$.

HOLECHEK, J.L.; VALDEZ, R. Evaluation of in vitro digestion for improving botanical estimates of mule deer fecal samples. Journal Mammalogy,v.66, n.3, p.574-577, 1985.

HOLECHEK, J.L.; VAVRA, M. The effect of slide and frequency observation numbers on the precision of microshitological analysis. Journal of Range Management, v.34, n.4, p.337-338, 1981.

HOLECHEK, J.L.; VAVRA, M.; PIEPER, R.D. Botanical composition determination of range herbivore diets: a review. Journal of Range Management, v.35, n.3, p.309-315, 1982 b.

JOHNSON, M.K. Frequency sampling for microscopic analysis of botanical compositions. Journal of Range Management, v.35, n.4, p.541-542, 1982.

JOHNSON, R.A.; WICHERN, D.W. Applied multivariate statistical analysis. 3.ed. New Jersey: Prentice Hall, 1992. $642 \mathrm{p}$.

LESLIE JR., D.M.; VAVRA, M.; STARKEY, E.E. et al. Correcting for differential digestibility in microhistological analyses involving common coastal forages of the pacific northwest. Journal of Range Management, v.36, n.6, p.730-732, 1983.
MUKHTAR, H.K.; HANSEN, R.M. Fiber effects on microhistological analysis. Journal of Range Management, v.36, n.4, p.477-478, 1983.

OOSTING, H.J. The study of plant communities. San Francisco: W.H. Freeman Co., 1956. 440p.

SAMUEL, J.; HOWARD, G.S. Disappearing forbs in microhistological analysis of diet. Journal of Range Management, v.36, n.1, p.132-133, 1983.

STATISTICAL ANALYSES SYSTEM - SAS. SAS/STAT user's guide. Version 6, 4.ed., v.1, Cary: 1990. 943p.

SCOTT, G.; DAHL, B.E. Key to selected plant species of Texas using plant fragments. Texas: The Museum. Texas Tech University, 1980. p.1-9. (Occasional Papers)

SLATER, J.; JONES, R.J. Estimation of the diets selected by grazing animals from microscopic analysis of faeces - A warning. Journal of Australian Institute of Agricultural Science, v.37, p.238-240, 1971.

SMITH, A.D.; SHANDRUK, L.J. Comparison of fecal, rumen and utilization methods for ascertaining pronghorn diets. Journal of Range Management, v.32, n.4, p.275-279, 1979.

SPARKS, D.R.; MALECHEK, J.C. Estimating percentage dry weight in diets using a microscopic technique. Journal of Range Management, v.21, n.4, p.264-265, 1968.

STROUP, W.W.; STUBBENDIECK, J. Multivariate statistical methods to determine chages in botanical composition. Journal of Range Management, v.36, n.2, p.208-212, 1983.

TILLEY, J.M.A.; TERRY, R.A. A two-stage technique for the in vitro digestion of forage crops. Journal British Grassland Society, v.18, n.2, p.104-111, 1963.

VAVRA, M.; HOLECHEK, J.L. Factors influencing microhistological analysis of herbivore diets. Journal of Range Management, v.33, p.371-374, 1980.

VOLTH, E.H.; BLACK, H.C. A histologic technique for determining feeding habits of small herbivores. Journal Wildlife Management, v.37, n.2, p.223-231, 1973.

WESTOBY, M.; ROST, G.R.; WEIS, J.A. Problems with estimating herbivore diets by microscopically identifying plant fragments from stomachs. Journal Mammalogy, v.57, n.1, p.167-172, 1978.

WILLIAMS, O. B. An improved technique for identification of plant fragments in herbivore feces. Journal of Range Management, v.22, n.1, p.51-52, 1969. 